\title{
ON RESTRICTED SYSTEMS OF HIGHER INDETERMINATE EQUATIONS *
}

BY

\section{E. T. BELL}

By two examples we shall illustrate a means for deriving arithmetical properties of certain restricted forms. So little being known concerning the arithmetic of forms of higher degree, it is reasonable to develop what is offered by analysis. The method exemplified is of wide scope and easy applicability, and may possibly, in addition to giving new properties of integers, yield also, on starting from the appropriate associated forms of the second degree, information regarding current problems. It is a natural extension to forms of higher degree of the classic applications of elliptic functions to special quadratic forms. The method being new, we shall give the analysis for the first example in some detail.

1. We take first a single restricted form

$$
u y^{s}+v x^{r}-x^{r} y^{s}
$$

in which $u, v, x, y$ are variable integers; $u, v$ are odd, $x, y$ even, and $r, s$ are constant odd positive integers. For $\alpha \geqq 1$ and $m$ odd and positive, we consider all the representations $(u, v, x, y)$ of $2^{\alpha} m$ in (1); or, what is the same, the totality of solutions $(u, v, x, y)$ of

$$
2^{\alpha} m=u y^{s}+v x^{r}-x^{r} y^{s},
$$

where $\alpha, m, r, s$ are constants, $m, u, v, r, s$ are odd, $x, y$ even, and $\alpha \geqq 1$. From all the $(u, v, x, y)$ we select two classes $C_{1}, C_{2}$ defined by
$\left(C_{1}\right)$ :
$x \gtreqless 0, \quad y>0$,
$u>x^{r}, \quad v<y^{s}$,
$\left(C_{2}\right)$ :
$x>0, \quad y \gtreqless 0$,
$u<x^{r}, \quad v>y^{s}$

and denote by $N_{i}\left(2^{\alpha} m\right)$ the number of $(u, v, x, y)$ belonging to $C_{i}$. It will be shown that the difference $N_{1}\left(2^{\alpha} m\right)-N_{2}\left(2^{\alpha} m\right)$ is a function of $\alpha$, $r, s$ and the divisors of $m$.

2. It is convenient to distinguish four cases:
$\left(A_{1}\right)$ :
$\alpha \equiv 0 \bmod r$
and
$\alpha \equiv 0 \bmod s$,
( $\left.A_{2}\right)$ :
$\alpha \equiv 0 \bmod r$
and
$\alpha \neq 10 \bmod s$,
( $\left.A_{3}\right)$ :
$\alpha \neq 0 \bmod r$
and
$\alpha \equiv 0 \bmod s$,
$\left(A_{4}\right)$ :
$\alpha$ 丰 $0 \bmod r$
and
$\alpha$ ⿻三丨 $0 \bmod s$.

* Presented to the Society, October, 1920. 
Let $\lambda_{j}(n)$ denote the sum of all those divisors of the positive integer $n$ which are $j$ th powers. Then, corresponding to the four cases we have:

$\begin{array}{ll}\left(A_{1}^{\prime}\right): & N_{1}\left(2^{\alpha} m\right)-N_{2}\left(2^{\alpha} m\right)=2^{\alpha-1}\left[\lambda_{s}(m)-\lambda_{r}(m)\right], \\ \left(A_{2}^{\prime}\right): & N_{1}\left(2^{\alpha} m\right)-N_{2}\left(2^{\alpha} m\right)=-2^{\alpha-1} \lambda_{r}(m), \\ \left(A_{3}^{\prime}\right): & N_{1}\left(2^{\alpha} m\right)-N_{2}\left(2^{\alpha} m\right)=2^{\alpha-1} \lambda_{s}(m), . \\ \left(A_{4}^{\prime}\right): & N_{1}\left(2^{\alpha} m\right)-N_{2}\left(2^{\alpha} m\right)=0,\end{array}$

all of which may be put in a single statement. To prove these results we transform (1) into an associated quadratic form, simultaneously replacing the conditions $\left(C_{1}\right),\left(C_{2}\right)$ by equivalent restrictions on the new variables.

3. The $(u, v, x, y)$ being as in $\S 1$, we see that $d^{\prime}, \delta^{\prime}, d^{\prime \prime}, \delta^{\prime \prime}$ defined by

$$
u=d^{\prime}, \quad v=\delta^{\prime}, \quad u-x^{r}=d^{\prime \prime}, \quad y^{8}-v=\delta^{\prime \prime},
$$

are odd positive integers provided that $(u, v, x, y)$ belongs to $C_{1}$. Let $(u, v, x ; y)$ belong to $C_{1}$. Then, since

$$
u y^{s}+v x^{r}-x^{r} y^{s}=u v+\left(u-x^{r}\right)\left(y^{s}-v\right),
$$

we may replace (2) by

$$
2^{\alpha} m=d^{\prime} \delta^{\prime}+d^{\prime \prime} \delta^{\prime \prime}
$$

in which $d^{\prime}, \delta^{\prime}, d^{\prime \prime}, \delta^{\prime \prime}$ are restricted to be odd positive integers subject to the conditions

$$
d^{\prime}-d^{\prime \prime}=x^{r}, \quad \delta^{\prime}+\delta^{\prime \prime}=y^{s}, \quad x \gtreqless 0,
$$

and, necessarily, $y>0$. It is easily seen that all those solutions of (2) belonging to $C_{1}$ are identical with all those of (4) which satisfy also (5). That is, (2) and $\left(C_{1}\right)$ define the same $(u, v, x, y)$ as do. (4) and (5). Hence $N_{1}\left(2^{\alpha} m\right)$ is equal to the number of solutions $\left(d^{\prime}, \delta^{\prime}, d^{\prime \prime}, \delta^{\prime \prime}\right)$ of (4) such that $d^{\prime}-d^{\prime \prime}$ is the $r$ th power of an integer 0 , and $\delta^{\prime}+\delta^{\prime \prime}$ the sth power of an integer $>0$, the $d^{\prime}, \delta^{\prime}, d^{\prime \prime}, \delta^{\prime \prime}$ being odd positive integers as defined.

Similarly, starting from

$$
u=d^{\prime}, \quad v=\delta^{\prime}, \quad x^{r}-u=d^{\prime \prime}, \quad v-y^{s}=\delta^{\prime \prime},
$$

where $d^{\prime}, \delta^{\prime}, d^{\prime \prime}, \delta^{\prime \prime}$ are odd positive integers, and $(u, v, x, y)$ belongs to $C_{2}$, we see that all the solutions of (2) belonging to $C_{2}$ are identical with all those of (4) which satisfr-also

$$
d^{\prime}+d^{\prime \prime}=x^{r}, \quad \delta^{\prime}-\delta^{\prime \prime}=y^{s}, \quad x>0, \quad y \gtreqless 0 ;
$$

that is, $N_{2}\left(2^{\alpha} m\right)$ is equal to the number of solutions $\left(d^{\prime}, \delta^{\prime}, d^{\prime \prime}, \delta^{\prime \prime}\right)$ of (4) which are such that $d^{\prime}+d^{\prime \prime}$ is the $r$ th power of an integer $>0$, and $\delta^{\prime}-\delta^{\prime \prime}$ the $s$ th power of an integer 0 . 
4. To evaluate $N_{i}\left(2^{\alpha} m\right)$ from this reduction to an associated quadratic form, we remark that if $n$ is the $k$ th power ( $k$ an integer) of an integer, $-n$ is also the $k$ th power of an integer when and only when $k$ is odd. Hence if $\varphi_{2 t-1}(x)$ denotes 1 or 0 according as $x$ is or is not the $(2 t-1)$ th power of an integer, $\varphi_{2 t-1}(x)$ is an even function of $x$, viz.,

$$
\varphi_{2 t-1}(x)=\varphi_{2 t-1}(-x) \text {. }
$$

Again, if $x, y$ are relatively prime,

$$
\varphi_{2 t-1}(x y)=\varphi_{2 t-1}(x) \varphi_{2 t-1}(y) ;
$$

and if $x, y, z, \cdots$ are integers, $r, s, t, \cdots$ odd positive integers, the value of

$$
\varphi_{r, 8, t}, \cdots(x, y, z, \cdots), \equiv \varphi_{r}(x) \varphi_{s}(y) \varphi_{t}(z) \cdots
$$

is unity when and only when simultaneously $x$ is an $r$ th power, $y$ is an sth power, $z$ is a $t$ th power, $\cdots$, and in every other case the function vanishes. That is, this function is even in each of its variables $x, y, z, \cdots$, and vanishes with each of $\varphi_{r}, \varphi_{s}, \varphi_{t}, \ldots$.

5. Using the values of $N_{i}\left(2^{\alpha} m\right)$ deduced in $\S 3$ from the associate (4), ve have now

$$
\begin{aligned}
& N_{1}\left(2^{\alpha} m\right)=\sum \varphi_{r, s}\left(d^{\prime}-d^{\prime \prime}, \delta^{\prime}+\delta^{\prime \prime}\right), \\
& N_{2}\left(2^{\alpha} m\right)=\sum \varphi_{r, s}\left(d^{\prime}+d^{\prime \prime}, \delta^{\prime}-\delta^{\prime \prime}\right),
\end{aligned}
$$

the $\sum$ extending to all the $d^{\prime}, \delta^{\prime}, d^{\prime \prime}, \delta^{\prime \prime}$ given by (4) as defined in $\S 3$. Let $f(x, y)$ denote any function which is even in each of its variables $x, y$. Then, the $\sum$ on the left being as just stated, that on the right extending to all positive divisors $d$ of $m$, there is the well-known result due to Liouville:

$$
\begin{aligned}
\sum\left[f\left(d^{\prime}-d^{\prime \prime} ; \delta^{\prime}+\delta^{\prime \prime}\right)-f\left(d^{\prime}+d^{\prime \prime},\right.\right. & \left.\left.\delta^{\prime}-\delta^{\prime \prime}\right)\right] \\
& =2^{\alpha-1} \sum d\left[f\left(0,2^{\alpha} d\right)-f\left(2^{\alpha} d, 0\right)\right] .
\end{aligned}
$$

Hence, substituting $\varphi_{r, s}(x, y)$ for $f(x, y)$, we have

(6) $N_{1}\left(2^{\alpha} m\right)-N_{2}\left(2^{\alpha} m\right)=2^{\alpha-1} \sum d\left[\varphi_{r, s}\left(0,2^{\alpha} d\right)-\varphi_{r, s}\left(2^{\alpha} d, 0\right)\right]$;

and since $d$ is odd,

$$
\varphi_{r}\left(2^{\alpha} d\right)=\varphi_{r}\left(2^{\alpha}\right) \varphi_{r}(d), \quad \varphi_{s}\left(2^{\alpha} d\right)=\varphi_{s}\left(2^{\alpha}\right) \varphi_{s}(d),
$$

so that (6) is equivalent to $\left(A_{1}^{\prime}\right), \cdots,\left(A_{4}^{\prime}\right)$ of $\S 2$.

6. In deriving such results the order of the steps is the reverse of that just completed. It will be sufficiently evident from the following example. The function $f(x, y)$ is as in $\S 5$, and all the letters, unless otherwise noted, represent positive integers. The origin of the fundamental $f$-identity is indicated in $\$ 8$. 
Consider the pair of equations

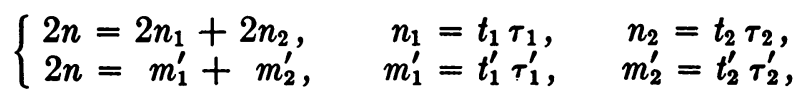

in which the $t, \tau$ are pairs of conjugate divisors, as indicated, and the $t$ 's are divisors whose conjugates are odd; $n_{1}, n_{2}$ are odd or even, $m_{1}^{\prime}, m_{2}^{\prime}$ are odd. Hence $t_{1}^{\prime}, \tau_{1}^{\prime}, t_{2}^{\prime}, \tau_{2}^{\prime}$ are odd, as also are $\tau_{1}, \tau_{2}$; and $t_{i}(i=1,2)$ is odd or even according as $n_{i}$ is odd or even, that is, $t_{i}$ and $n_{i}$ are of the same parity. Then, the $\sum$ on the left referring to all $t, \tau, m_{2}^{\prime}$ which satisfy (7) for $n$ given, that on the right to all pairs of conjugate divisors $t, \tau$ of $n(=t \tau)$, we have

$$
\begin{aligned}
\sum\left[(-1)^{t_{2}}\{\right. & \left.f\left(2 t_{2}+\tau_{1}, \tau_{2}\right)-f\left(2 t_{2}-\tau_{1}, \tau_{2}\right)\right\} \\
& \left.-(-1)^{t_{1}}\left\{f\left(\tau_{2}, 2 t_{2}+\tau_{1}\right)-f\left(\tau_{2}, 2 t_{2}-\tau_{1}\right)\right\}\right] \\
& +2 \sum\left(-1 \mid \tau_{1}^{\prime}\right) \xi\left(m_{2}^{\prime}\right) f\left(t_{1}^{\prime}, \tau_{1}^{\prime}\right) \\
& =\sum\left[\sum_{h=1}^{t}\left\{f(2 h-1, \tau)-(-1)^{n} f(\tau, 2 h-1)\right\}\right],
\end{aligned}
$$

where $\left(-1 \mid \tau_{1}^{\prime}\right)$ is Jacobi's extension of Legendre's symbol, and $\xi\left(m_{2}^{\prime}\right)$ one fourth the number of representations of $m_{2}^{\prime}$ as a sum of two squares. This identity indicates, on replacing $f(x, y)$ by $\varphi_{r_{,}}(x, y)$, the substitutions for transformng (7) into four higher forms, also what restrictions are to be imposed on the latter.

7. For this purpose we consider the first partial sum on the left (after replacing $f$ by $\varphi$ ),

$$
\sum(-1)^{t_{2}} \varphi_{r_{,},}\left(2 t_{2}+\tau_{1}, \tau_{2}\right) \text {. }
$$

Here the $\varphi$-function $=1$ when and only when integers $x, y$ exist such that

$$
2 t_{2}+\tau_{1}=x^{r}, \quad \tau_{2}=y^{2} .
$$

In every other case the value is zero. Hence we replace the first of the equations (7) by

$$
2 n=2 v x^{r}+2 u y^{2}-4 u v,
$$

the substitution converting it into (8) being

$$
t_{2}=u, \quad \tau_{1}=x^{r}-2 u, \quad t_{1}=v, \quad \tau_{2}=y^{s} .
$$

By translating the conditions upon the $t, \tau$ into terms of the new variables, we get the restrictions to which (8) is subjected:

$\left(C_{1}\right): \quad x, y$ odd $>0 ; u, v$ odd or even, $>0 ; x^{r}>2 u$.

Further subdividing $C_{1}$ according as $u$ is even or odd, we have two subclasses, $\left(C_{10}\right): x, y$ odd, $>0 ; u$ even, $>0$; $v$ odd or even, $>0 ; x^{r}>2 u$; $\left(C_{11}\right): x, y$ odd, $>0 ; u$ odd, $>0$; $v$ odd or even, $>0 ; x^{r}>2 u$. 
Let $N_{i j}(2 n)$ denote the number of solutions of (8) which belong to $C_{i j}$. Then, as in the first example, we have

$$
\sum(-1)^{t_{2}} \varphi_{r_{\text {, }}}\left(2 t_{2}+\tau_{1}, \tau_{2}\right)=N_{10}(2 n)-N_{11}(2 n) .
$$

The third partial sum also refers to (8), the substitution being

$$
t_{2}=v, \quad \tau_{1}=y^{s}-20, \quad t_{1}=u, \quad \tau_{2}=x^{r},
$$

and the corresponding classes

$\left(C_{30}\right): \quad x, y$ odd, $>0 ; u$ even, $>0 ; v$ odd or even, $>0 ; y^{*}>2 v$;

$\left(C_{31}\right): x, y$ odd, $>0 ; u$ odd, $>0 ; v$ odd or even, $>0 ; y^{*}>2 v$,

whence $\sum(-1)^{t_{1}} \varphi_{r_{,}}\left(\tau_{2}, 2 t_{2}+\tau_{1}\right)=N_{30}(2 n)-N_{31}(2 n)$.

The second partial sum refers to the equation

$$
2 n=-2 v_{1} x_{1}^{r}+2 u_{1} y_{1}^{s}+4 u_{1} v_{1},
$$

the substitution and the classes being, with a notation similar to that for the preceding cases,

$$
t_{2}=u_{1}, \quad \tau_{1}=2 u_{1}-x_{1}^{r}, \quad t_{1}=v_{1}, \quad \tau_{2}=y_{1}^{\mathrm{r}} ;
$$

$\left(C_{20}\right): x_{1}, y_{1}$ odd, $x_{1} \gtrless 0, y_{1}>0 ; u_{1}$ even, $>0$; $v_{1}$ odd or even, $>0 ; x_{1}^{r}<2 u_{1}$;

$\left(C_{21}\right): x_{1}, y_{1}$ odd, $x_{1} \gtrless 0, y_{1}<0 ; u_{1}$ odd, $>0$; $v_{1}$ odd or even, $>0 ; x_{1}^{r}<2 u_{1}$;

whence

$$
\sum(-1)^{t_{2}} \varphi_{r, 8}\left(2 t_{2}-\tau_{1}, \tau_{2}\right)=N_{20}(2 n)-N_{21}(2 n) .
$$

The fourth partial sum refers to

$$
2 n=2 v_{2} x_{2}^{r}-2 u_{2} y_{2}^{s}+4 u_{2} v_{2},
$$

the substitution and classes being

$$
t_{2}=v_{2}, \quad \tau_{1}=2 v_{2}-y_{2}^{\mathrm{n}}, \quad t_{1}=u_{2}, \quad \tau_{2}=x_{2}^{r} ;
$$

$\left(C_{40}\right): x_{2}, y_{2}$ odd, $x_{2}>0, y_{2} \gtrless 0 ; u_{2}$ even, $>0 ; v_{2}$ odd or even, $>0 ; y_{2}^{s}<2 v_{2}$;

$\left(C_{41}\right): x_{2}, y_{2}$ odd, $x_{2}>0, y_{2} \gtrless 0 ; u_{2}$ odd, $>0 ; v_{2}$ odd or even, $>0 ; y_{2}^{\prime}<2 v_{2}$;

whence

$$
\sum(-1)^{t_{1}} \varphi_{r, 8}\left(\tau_{2}, 2 t_{2}-\tau_{1}\right)=N_{40}(2 n)-N_{41}(2 n) .
$$

The fifth partial sum refers to the equation

$$
2 n=x_{3}^{r} y_{3}^{s}+u_{3}^{2}+v_{3}^{2},
$$

for which the substitution and classes are

$$
t_{1}^{\prime}=x_{3}^{r}, \quad \tau_{1}^{\prime}=y_{3}^{\prime}, \quad m_{2}^{\prime}=u_{3}^{2}+v_{3}^{2} ;
$$

$\left(C_{50}\right): x_{3}, y_{3}$ odd, $>0 ; u_{3}, v_{3}$ of opposite parities, ऐ $0 ; y_{3} \equiv 1 \bmod 4$;

$\left(C_{51}\right): x_{3}, y_{3}$ odd, $>0 ; u_{3}, v_{3}$ of opposite parities, ऐ $0 ; y_{3} \equiv-1 \bmod 4$;

Trans. Am. Math. Boc. 32. 
and we have

$$
2 \sum\left(-1 \mid \tau_{1}^{\prime}\right) \xi\left(m_{2}^{\prime}\right) \varphi_{r, 8}\left(t_{1}^{\prime}, \tau_{1}^{\prime}\right)=\frac{1}{2}\left[N_{50}(2 n)-N_{51}(2 n)\right] .
$$

To evaluate the right side of the identity we denote by $[x]$ the greatest integer which does not exceed $x$, and by $t_{j}$ a positive divisor of $n$ whose conjugate; $\tau_{j}$, is a $j$ th power. Then, the $\sum$ on the right referring to all $t_{r}, t_{s}$, we have at once

$$
\begin{aligned}
\sum\left[\sum_{h=1}^{t}\left\{\varphi_{r, s}(2 h-1, \tau)-(-1)^{n} \varphi_{r, s}(\tau, 2 h-1)\right\}\right] & \left.=\sum\left\{\sqrt[r]{2 t_{s}-1}\right]-(-1)^{n}\left[\sqrt[f]{2 t_{r}-1}\right]\right\} .
\end{aligned}
$$

Combining all these results, we get for the four equations (8), (9), (10), (11) the following syzygy between the number of solutions belonging to the classes $C_{i j}$ :

$$
\begin{aligned}
2\left\{N_{10}(2 n)-N_{11}(2 n)-\right. & N_{20}(2 n)+N_{21}(2 n)-N_{30}(2 n)+N_{31}(2 n) \\
+ & N_{40}(2 n)- \\
= & \left.N_{41}(2 n)\right\}+N_{50}(2 n)-N_{51}(2 n) \\
& \left.2 \sum\left[\sqrt[5]{2 t_{s}-1}\right]-(-1)^{n}\left[\sqrt[3]{2 t_{r}-1}\right]\right\} ;
\end{aligned}
$$

and this may be regarded as a relation between the numbers of simultaneous representations of $2 n$ in the system of forms

$$
\begin{gathered}
2 v x^{r}+2 u y^{s}-4 u v ; \quad-2 v_{1} x_{1}^{r}+2 u_{1} y_{1}^{s}+4 u_{1} v_{1} ; \\
2 v_{2} x_{2}^{r}-2 u_{2} y_{2}^{s}+4 u_{2} v_{2} ; \quad x_{3}^{r} y_{3}^{r}+u_{3}^{2}+v_{3}^{2},
\end{gathered}
$$

subject to the given restrictions. In both examples the number of representations in each class is obviously, from the associate, finite.

8. The identity in $\S 6$ is one paraphrase of the equation of three terms in elliptic functions; Liouville's in $\$ 5$ may be derived from the same source, as shown in papers presented to the Society in 1918. By the methods of those papers combined with the present, similar results for systems of any number of forms in any number of indeterminates may be found.

UNIVERSITY OF WASHINGTON, Seattre, Wash. 